Irene Becci, Marian Burchardt, José Casanova,
Topographies of Faith. Religion in Urban Spaces

Leiden, Brill, 2013, 235 p.

Mariachiara Giorda

(2) OpenEdition

Journals

Édition électronique

URL : http://journals.openedition.org/assr/26299

DOI : 10.4000 /assr.26299

ISSN : $1777-5825$

Éditeur

Éditions de l'EHESS

Édition imprimée

Date de publication : 31 décembre 2014

Pagination : 123

ISBN : 978-2-7132-2467-6

ISSN : 0335-5985

Référence électronique

Mariachiara Giorda, «Irene Becci, Marian Burchardt, José Casanova, Topographies of Faith. Religion in Urban Spaces », Archives de sciences sociales des religions [En ligne], 168 | 2014, mis en ligne le 02 avril 2015, consulté le 22 septembre 2020. URL : http://journals.openedition.org/assr/26299 ; DOI : https:// doi.org/10.4000/assr.26299

Ce document a été généré automatiquement le 22 septembre 2020.

(c) Archives de sciences sociales des religions 


\section{Irene Becci, Marian Burchardt, José Casanova, Topographies of Faith. Religion in Urban Spaces}

Leiden, Brill, 2013, 235 p.

Mariachiara Giorda

\section{RÉFÉRENCE}

Irene Becci, Marian Burchardt, José Casanova, Topographies of Faith. Religion in Urban Spaces, Leiden, Brill, 2013, 235 p. 
1 Une femme qui arrive en gare d'une ville européenne, Rome par exemple, pourrait s'étonner de voir les multiples signes d'un pluralisme religieux: synagogues, mosquées, églises évangéliques, paroisses catholiques, temples bouddhistes, gurudwara.

2 En renversant la célèbre image du paysan français qui abandonne son catholicisme à la Gare de Montparnasse, suggérée par Le Bras (et citée dans cet ouvrage à la page 3), le pluralisme religieux contemporain profite des nouveaux effets de la sécularisation dans les villes; la complexité et la grande diversité sont les mots-clés utiles pour lire les nouveaux contextes urbains. L'ouvrage dirigé par Becci, Burchardt et Casanova est une manière intéressante de réfléchir aux

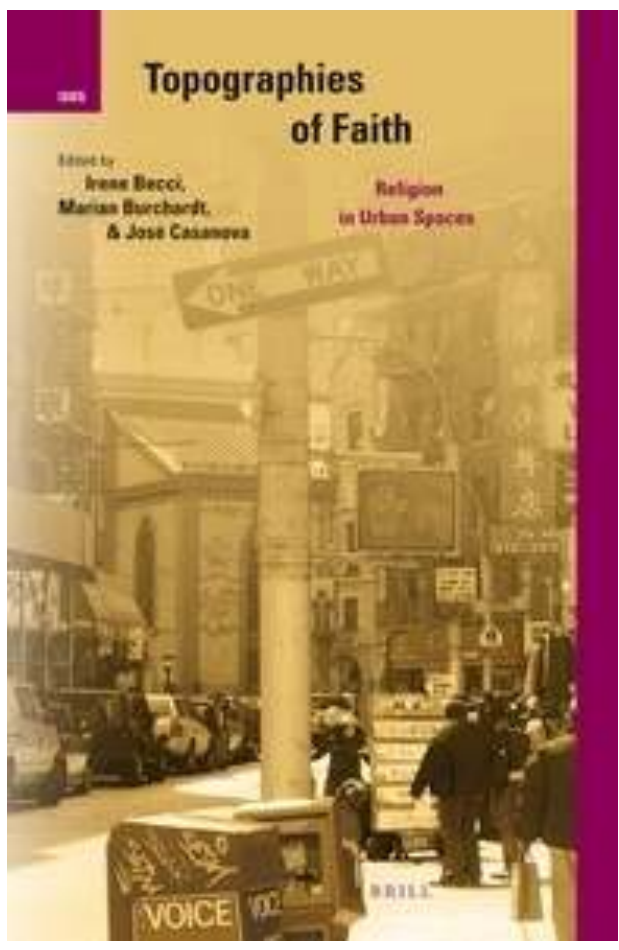
relations entre changements religieux et innovations urbaines.

3 Les données sociogéographiques con-firment l'urgence de nouvelles études sur les espaces urbains: selon le United Nations Report, à partir de 2008, plus de $50 \%$ de la population mondiale vit dans des aires urbaines et le United Nations Population Fund affirme que $60 \%$ de la population mondiale, environ 5 billions de personnes, vivront en ville d'ici 2030 (2007 State of World Population (UNFPA) and World Population Prospects). Les taux de chômage, la pauvreté et les inégalités sociales - ainsi que les processus intensifs d'urbanisation, globalisation et l'industrialisation - ont augmenté. Il devient donc urgent de connaître le rôle que joue la religion pour soulager les problèmes sociaux, augmenter le bien-être social et construire une justice sociale : la religion fait partie de la culture urbaine et, d'une manière ou d'une autre, aborde certains des défis d'une société caractérisée par de fortes inégalités et hétérogénéités.

Cela est suffisant pour souligner l'importance d'une nouvelle réflexion sur les espaces urbains et les relations sociales, culturelles et religieuses qui naissent en leur sein. Comme les auteurs du livre le disent, les villes peuvent (encore) servir de « loupe pour la théorie sociale " (S. Sassen, The Global City: New York, London, Tokyo, Princeton, Princeton University Press, 2001, p.4) et parmi les dynamiques urbaines, les changements religieux sont (encore) un sujet intéressant, en dépit ou en tant que conséquence du processus de sécularisation et de modernisation.

5 En prenant comme exemples plusieurs villes dans le monde, les auteurs estiment insuffisant le paradigme de la «dichotomie de la civilisation » (par exemple dans les termes de l'opposition entre Est/Ouest ou sociétés riches/pauvres, en référence à un monde séculier opposé à un monde religieux, R. Inglehart, P. Norris, Sacred and Secular : Religion and Politics Worldwide, Cambridge University Press, 2004), le paradigme de «l'exception » (Europe séculière, mais États-Unis religieux, Peter Berger, Grace Davie, and Effie Fokas. Religious America, Secular Europe? A Theme and Variations, Farnham/ Burlington: Ashgate, 2008), ou encore le paradigme de l'opposition entre le séculier et 
le religieux en tant que deux domaines séparés. Ce dernier binôme représente un tournant essentiel pour comprendre la vision du livre et de quelle manière la séculariation et la religion sont traitées.

6 Le mot "séculier» dérive du latin saeculum, c'est-à-dire "le temps présent ", et les termes sécularisation et sécularisme sont entrés dans le débat politique et culturel au cours du xiX siècle. Dans cette perspective, le désenchantement du monde (Marcel Gauchet, Le Désenchantement du monde. Une histoire politique de la religion, Gallimard, Paris, 1985) signifiait aussi l'émancipation de la culture et de l'éducation du contrôle ecclésiastique traditionnel.

Cependant, comme tout autre concept, celui de sécularisation ou de "sécularisme " n'est pas universel ni an-historique et il a été repensé et reformulé (M. Burchardt, M. Wohlrab-Sahr, «Multiple Secularities: Towards a Cultural Sociology of Secular Modernities ", in Comparative Sociology, 11, 2012, p. 875-909), avec une Umbesetzung - à la Blumenberg - comme on peut le comprendre dans les pages de l'introduction et en particulier dans l'article de Casanova. Avec une position forte à l'égard de l'explosion de la catégorie de "post-séculier» (C. Baker, J. Beaumont, Postsecular Cities: Space, Theory and Practice, London [etc.], Continuum, 2011, p. 17, mais aussi Jürgen Habermas, « Notes on Post-Secular Society », New Perspectives Quarterly, 25, n. 4, 2008, p. 17-29), les auteurs du livre utilisent "sécularisation" en tant que processus dynamique et toujours en cours, qui a amené à différents modèles de séparation entre l'État séculier et la religion, à différentes approches de leurs implications en termes d'éthique, de lois, de sciences, de philosophie, et d'économie, et pour ce qui concerne l'autonomie des sphères institutionnelles par rapport aux institutions religieuses; et encore dans le même temps, à une nouvelle possibilité pour les communautés religieuses de s'exprimer dans l'espace publique, liée à la liberté religieuse et de culte. En d'autres termes, le sécularisme contemporain n'est pas la disparition de la religion. De plus, la religion est un produit de la modernité, puisque nul discours concernant le moderne et la modernité ne peut oublier la religion : la dichotomie entre séculier/religieux est déjà un produit de la modernité séculière et la simple opposition entre ces deux termes est fausse (Casanova, p. 121). Si saeculum est le monde et si la religion fait partie de la culture et de l'histoire des individus et des communautés (selon la perspective des sciences des religions), la religion fait également partie du monde et, par conséquent, ne peut être opposée au séculier. En ce sens, les espaces urbains étudiés par les auteurs du livre (Berlin, Cape Town, Johannesburg, Mumbai, New Delhi, New York, Singapour, Istanbul et Lagos) sont très éloignés de la technopolis, la ville séculière privée de religion qu'avait évoquée Harvey Cox en 1965, quand la ville moderne apparaissait en mesure de remodeler radicalement la société humaine et l'Église. Au contraire, plusieurs villes sont des centres vivants de l'urbanisation religieuse et sont devenues la réalisation de sociétés radicalement plurielles du point de vue des religions, de la foi, des croyances, à travers la présence de communautés et de lieux religieux qui occupent l'espace publique. Comme Kim Knott le suggérait (Kim Knott, The Location of Religion: A Spatial Analysis, Equinox Publishing Ltd., 2005), les villes sont un réseau d'hommes et de lieux, une constellation complexe d'endroits, personnes, bâtiments, institutions, biens, capitaux, idées, intersections de routes, relations, diasporas et transactions et les religions ont un espace important, soit physique soit psychologique et un rôle d'interconnexion dans ces réseaux urbains. 
8 La religion est un facteur de transformation de la ville, qui s'insère au sein d'un processus de coexistence et de complémentarité entre religieux et séculier, en ce qui concerne leurs visions du monde et leurs pratiques. Ce processus peut être également interprété en termes de visibilité/invisibilité, inclusion/exclusion, public/privé. Le fait que de nombreuses villes soient des centres d'innovation religieuse nous oblige à mettre en discussion les hypothèses sur les effets de totale sécularisation, qui seraient déterminés par l'urbanisation. Le changement urbain survenu lors du passage à l'ère post-industrielle semble, en effet, remodeler les différences religieuses, plutôt que les éclipser. La sécularisation et la modernisation ont contribué à créer un nouveau rôle privé et public de la religion dans les villes.

Ce livre, dont le résultat est fondé sur des séminaires et des ateliers (p. VII) d'un groupe de spécialistes, se concentre sur trois thèmes principaux, fils rouge des sections du livre: "Religious innovation of urban contexts »; "Urban dynamics of migration, religious diversity and transnational religion » ; « Religion, economic Inequalities and Social Exclusion ».

Dans la première section, on retrouve quatre essais: Murat Es, dans son Alevis in Cemevis: Religion and Secularism in Turkey, enquête sur la reconstitution de l'Alévisme en tant que religion séculière enracinée dans un contexte urbain (Istanbul, auprès du l'okmeydani Cemevi), à travers les politiques internationales de reconnaissance et les relations avec la communauté musulmane sunnite turque. Somalis in Johannesburg: Muslim Transformations of the City de Samadia Sadouni est une contribution aux études sur la diversité au sein de l'islam, à travers l'analyse de l'impact religieux et social d'un groupe de réfugiés somaliens sur la ville, en termes de l'organisation de la solidarité (les Somalis ont formé leurs communautés congrégationales qui supportent les pauvres et les marginaux) et de lutte contre la xénophobie. Dans Urban Aspirations in Mumbai and Singapore, Peter van der Veer distingue deux niveaux d'analyse qu'il traite trop souvent séparément: l'économique et le spirituel/culturel. Le voyage au sein de l'identité complexe de Mumbai et Singapour est une tentative - non seulement aboutie, mais paradigmatique - de réflexion sur le sentiment d'appartenance, les (auto)représentations et les aspirations de deux villes " globales ». Dans Excarnation and the City; The Tower of Silence Debates in Mumbai, Leilah Vevaina reconstruit, à partir des questions suscitées par la pratique funéraire des Parsi, l'histoire du rapport entre une métropole et les exigences pratiques d'un groupe minoritaire, en analysant les conséquences religieuses, sociales, politiques et économiques : aujourd'hui la pratique de l'« excarnation " (les corps morts sont laissés aux oiseaux et au soleil) n'est plus acceptable pour des raisons écologiques, hygiéniques et sociales et la tour sacrée de cette pratique est trop grande et trop centrale par rapport aux exigences de la ville.

La deuxième section ouvre sur l'essai de Synnove Bendixsen, Connecting the Local, National and Transnational Powers of Religious Youth Organisation in Berlin. En se fondant sur l'observation de la vie quotidienne des membres d'une organisation de jeunes musulmans allemands, l'article offre un exemple de la formation du sentiment d'identité collective grâce à la transmission des savoirs et des pratiques religieuses à plusieurs niveaux: local, national et transnational. Religious associations, Religious Innovations and Denominational Identities in Contemporary Global Cities représente une réflexion brillante de José Casanova sur l'importance croissante de la religion dans les processus d'urbanisation caractérisant les villes partout dans le monde. Le sociologue propose à nouveau, dans ce texte, certains thèmes qui lui sont familiers : dans le cadre de la modernité et de la globalisation, il reprend le binôme séculier/religieux, le lien 
entre la liberté religieuse et les droits de l'homme, la relation entre autorité, religions et démocraties. The Geopolitics of Religious Spatiality and Falun Gong's Campaign in New York de Weishan Huang est une enquête sur les stratégies d'occupation des espaces (en particulier à Manhattan) par la FLG, un mouvement religieux chinois qui naît au début des années 1990 et qui a été exilé du pays d'origine après 1999.

Dans la troisième section, Irene Becci nous présente, dans Religious Involvements in a Post-Socialist Urban Space in Berlin, un exemple de la manière dont une communauté religieuse (une congrégation baptiste) est aussi un acteur social : cette enquête montre les stratégies que le groupe met en pratique en sollicitant l'espace d'une ville telle que Berlin pour s'intégrer et obtenir un espace, une visibilité, pour être acceptés des habitants de la ville et pour attirer de nouveaux fidèles.

Marian Burchardt, dans Belonging and Success: Religious Vitality and the Politics of Urban Space in Cape Town, propose d'avoir recours aux catégories d'appartenance et de succès en tant qu'instruments de lecture pour analyser les liens entre l'espace urbain et l'espace religieux. Cette analyse s'articule, dans le cas présent, autour d'une recherche menée dans certaines Églises chrétiennes de Cape Town [ou Ville du Cap]. Porous Boundaries: Hindu-Muslim Demarcation and Crossings in New Delhi d'Ajay Gandhi est une enquête concernant les pratiques de groupes religieux habitant une même ville, afin d'établir une comparaison et également repérer un "air de famille» dans la vie quotidienne des membres d'une communauté hindoue et musulmane. Enfin, «Exit » and "inclusion »: The Changing Paradigm of Pentecostal Expression in the Nigerian Public Space, de Godwin Onuoha, examine les expressions (aspirations, imagination, sentiments d'identité et d'appartenance) de certains mouvements pentecôtistes et charismatiques au Nigéria, dans les termes d'exclusion et d'inclusion dans l'espace public.

Pour une lecture critique du livre, on peut cependant remarquer certaines limites et absences : la question de la distinction entre lieu et espace (Becci cite M. de Certeau, p.149) n'est pas suffisamment développée et on ne retrouve pas une analyse approfondie du concept de «spatial turn» (p.13) - tel qu'il a été traité par les spécialistes des sciences humaines - et en particulier de ses conséquences pour les sciences historico-religieuses. Du point de vue de l'architecture et des urban studies, l'un des sujets les plus intéressants (mais insuffisamment traité dans l'ouvrage) est l'occupation de l'espace urbain à travers différentes tactiques et stratégies, ainsi que la distribution de l'activité spirituelle à travers la ville et la manière dont une telle distribution donne naissance à une réalité urbaine caractérisée par des déplacements et des adaptations. En lien avec cela, des règles publiques deviennent inévitables pour la planification de l'avenir, et l'exigence d'être des villes durables est de plus en plus liée à l'éthique et aux systèmes de valeur que les religions mettent en évidence. Néanmoins, on peut dire que ces absences sont les conséquences normales des limites d'un projet aussi vaste.

En conclusion, cet ouvrage apporte une importante contribution non définitive au débat concernant la religion et le sécularisme dans les espaces urbains contemporains et suggère que le thème doit être encore étudié, avec de nouveaux cas et une perspective encore plus interdisciplinaire. On espère que ce projet se poursuivra et impliquera des spécialistes provenant aussi de disciplines autres que la sociologie et l'anthropologie, afin de discuter la complexité de l'objet et - comme les auteurs le suggèrent dans l'introduction - de vérifier si notre vocabulaire est approprié à la nature fluide de la religion et du sécularisme. 